\section{Sudden death in a young patient with atrial fibrillation}

\author{
María Tamargo, \\ María Ángeles Espinosa, \\ Víctor Gómez-Carrillo, Miriam Juárez, \\ Francisco Fernández-Avilés, \\ Raquel Yotti

\begin{abstract}
Inherited Cardiovascular Disease
Program, Cardiology Department, Gregorio Marañón Hospital, Madrid, Spain
\end{abstract}

\begin{abstract}
Sudden cardiac death (SCD) in young patients without structural heart disease is frequently due to inherited channelopathies such as long QT syndrome (LQTS), Brugada syndrome or Catecholaminergic polymorphic ventricular tachycardia. Accordingly, the addition of genetic testing to clinical data may be useful to identify the cause of the sudden death in this population. Mutations in the KCNQ1 encoded Kv7.1 channel are related to type 1 LQTS, familial atrial fibrillation (AF), short QT syndrome, and SCD. We present a clinical case where the presence of $\mathrm{AF}$ after resuscitation in a young man with cardiac arrest was the key clinical data to suspect an inherited disorder and genetic testing was the main determinant for identifying the cause of the cardiac arrest. The KCNQ1 p.Arg231His mutation explained the combined phenotype of AF and susceptibility to ventricular arrhythmias. The case highlights the importance of continued research in genetics and molecular mechanisms of channelopathies.
\end{abstract}

\section{Introduction}

Sudden cardiac death (SCD) is a major contributor to cardiovascular mortality being responsible for approximately $25 \%$ of total cardiovascular deaths in the world with a global estimated incidence of 4 million cases per year. ${ }^{1}$ \{Priori, 2015 \#12@@authoryear\}\{Priori,2015 \#12@@hidden\}\{Priori, 2015 \#1\}\{Priori, 2015 \#3\}\{Priori, 2015 \#1\} Although ischaemic heart disease accounts for the majority of cases, in patients under 40 years old, inherited structural or purely arrhythmogenic cardiac disorders such as long QT syndrome (LQTS), Brugada syndrome (BS) or Catecholaminergic polymorphic ventricular tachycardia (CPVT) are the main cause of SCD. ${ }^{2}$

\section{Case Report}

A 22-year-old man suffered a sudden cardiac arrest without previous symptoms while he was at rest, waiting for a subway train. Cardiopulmonary resuscitation was immediately started using an Automated External Defibrillation that identified the presence of ventricular fibrillation and delivered a shock. Return of spontaneous circulation was achieved after three attempts, being atrial fibrillation (AF) the patient's rhythm at this point (Figure 1).

He was admitted to our Cardiovascular Intensive Care Unit and therapeutic hypothermia was performed over a period of $24 \mathrm{~h}$. After completing hypothermia, rewarming, and another $24 \mathrm{~h}$ of controlled normothermia the patient awakened with no residual neurologic damage. During hospitalization, structural heart disease was ruled out with a transthoracic echocardiogram, coronary angiography and a cardiac magnetic resonance. Since AF persisted, an elective electrical cardioversion was successfully performed at day four after admission. 12-lead electrocardiogram (ECG) showed a normal QT interval with a corrected QT (QTc) of $423 \mathrm{~ms}$. Continuous ECG monitoring showed no electrical abnormalities after returning to sinus rhythm (Figure 2). An exercise test on treadmill showed no ventricular ectopics or ventricular arrhythmias (VA). QTc interval shortened accordingly and at $4 \mathrm{~min}$ of recovery remained $<460 \mathrm{~ms}$. Flecainide test was also normal. An implantable cardioverter defibrillator (ICD) was implanted as secondary prevention.

The patient was an exchange student with no previous relevant medical records and all his family lived overseas. The family pedigree was recorded. There was no known history of SCD, unexplained syncope or AF in the family. His mother had died due to a non-cardiac disease and his father and two sisters were alive and apparently healthy. A paternal cousin had also died but the patient was not aware of the details (Figure 3).

Next generation sequencing of a set of 76 cardiac disease-related genes identified the heterozygous KCNQ1 missense variant p.Arg231His (R231H) in exon 5. This variant results in the replacement of the normal arginine codon (CGC) with a histidine codon (CAC) at the amino acid position 231 in the S4 domain of the ion channel. It has been previously described in patients with type 1 LQTS (LQT1) and interfamilial early-onset $\mathrm{AF}^{3-7}$

Treatment with beta blockers was start-
Correspondence: María Angeles Espinosa Castro, Inherited Cardiovascular Disease Program, Cardiology Department, Gregorio Marañón Hospital, Dr. Esquerdo, 46, 28007, Madrid, Spain.

Tel.: +34.91.586.82.90

E-mail: mariaangeles.espinosa@salud.madrid.org

Key words: KCNQ1; mutation; channelopathy; sudden cardiac death; atrial fibrillation.

Contributions: MT, acquisition and interpretation of data for the work, ensuring that questions related to the accuracy or integrity of any part of the work is appropriately investigated and resolved; MAE, conception of the work, critical revision of the intellectual content, final approval of the version to be published, ensuring that questions related to the accuracy of any part of the work is appropriately investigated and resolved; VG-C, acquisition and interpretation of data for the work; MJ, acquisition of data for the work; FF-A, final approval of the version to be published; RY, critical revision of the intellectual content, final approval of the version to be published, ensuring that questions related to the accuracy of the work is appropriately investigated.

Conflict of interest: the authors declare no potential conflict of interest.

Funding: Fondo Europeo de Desarrollo Regional, Instituto de Salud Carlos III, Madrid, Spain.

Conference presentation: this case was accepted for poster presentation at the Heart Failure 2016 annual congress of the Heart Failure Association of the ESC in Florence, Italy.

Received for publication: 24 September 2016. Revision received: 26 July 2017.

Accepted for publication: 14 August 2017.

This work is licensed under a Creative Commons Attribution NonCommercial 4.0 License (CC BY-NC 4.0).

CC Copyright M. Tamargo et al., 2017

Licensee PAGEPress, Italy

Cardiogenetics 2017; 7:6304

doi:10.4081/cardiogenetics.2017.6304

ed (Nadolol $40 \mathrm{mg}$ twice a day) and genetic counselling given. Unfortunately, we could not study any member of the family so the current status of the relatives is not available. We strongly recommended him to advise all his first-degree relatives for genetic screening and clinical cardiac evaluation with at least a basal ECG, treadmill exercise test and Holter monitoring in order to assess arrhythmic risk in the family and provide preventive care. 


\section{Discussion}

SCD in young patients is a dramatic event and identifying the final cause might be challenging. Once ischaemic heart disease and structural cardiomyopathy have been ruled out, the aetiology still remains unknown in up to $50 \%$ of the cases. ${ }^{2}$ In this scenario, an inherited arrhythmogenic disorder should always be considered.

In our case, the patient presented normal ECG intervals and provocative tests were negative for LQTS, BS and CPVT. However, he had lone persistent AF which is a rare finding in a young patient with a structural normal heart. The presence of the KCNQ1 R231H variant was the key point to reach a final diagnosis.

KCNQ1 gene codes for the pore-forming a-subunit Kv7.1 which is a main component of the macromolecular voltagegated potassium channel protein that carries the slowly activated delayed rectifier potassium current $\left(\mathrm{I}_{\mathrm{Ks}}\right)$. $\mathrm{I}_{\mathrm{Ks}}$ plays an important role in human cardiac repolarization, particularly at fast rates and when sympathetic tone increases. $\mathrm{I}_{\mathrm{Ks}}$ has a small amplitude under basal conditions so it is not a major contributing factor to normal repolarization but it is highly recruited when the action potential duration (APD) prolongs and during $\beta$-adrenergic stimulation. 89 Therefore, $\mathrm{I}_{\mathrm{Ks}}$ protects against excessive lengthening of APD and is the main responsible for the adaptation of the APD to heart rate. ${ }^{10}$

LQT1 is caused by loss-of-function mutations in KCNQ1 gene. The subsequent $\mathrm{I}_{\mathrm{Ks}}$ current decrease leads to a delayed repolarization, QT interval prolongation and enhanced susceptibility to ventricular arrhythmias, notably torsade de Pointes that occur mainly at exercise and during sympathetic stress. ${ }^{11}$ Contrarily, gain of function mutations of the KCNQ1 gene have also been described, resulting in shortening of the APD and the refractory period. Gain of function mutations have been associated with sinus bradycardia, SQTS and familial AF. ${ }^{7,12}$

The KCNQ1 R231H variant is considered a pathogenic mutation. It was first described by Napolitano in a patient with LQTS, though there is no information available regarding the QT interval or the exact phenotype. ${ }^{3}$ Posteriorly, other 7 non-related families and 19 carriers have been reported..$^{4-7}$ Interestingly, early-onset AF was the main phenotype in the families whereas most carriers (14) had a normal QT interval. 2 gene carriers presented upper limit QTc: a newborn male with fetal bradycardia $^{6}$ and a patient with AF. ${ }^{7}$ The 3 remaining patients reviewed are described as having long QT, though in one case long
QT was unmasked during infusion of epinephrine $^{7}$ and information about the QT interval or the exact phenotype is lacking in the other case. ${ }^{5}$ The last patient was a 1 year-old female with LQTS (QTc $479 \mathrm{~ms}$ ) and $\mathrm{AF}^{4,7}$ Moreover, in a family with early onset $\mathrm{AF}$, a male gene carrier experienced SCD at rest (while sleeping) having normal QT, just as the case of our patient.?

Even if initially the $\mathrm{R} 231 \mathrm{H}$ mutation

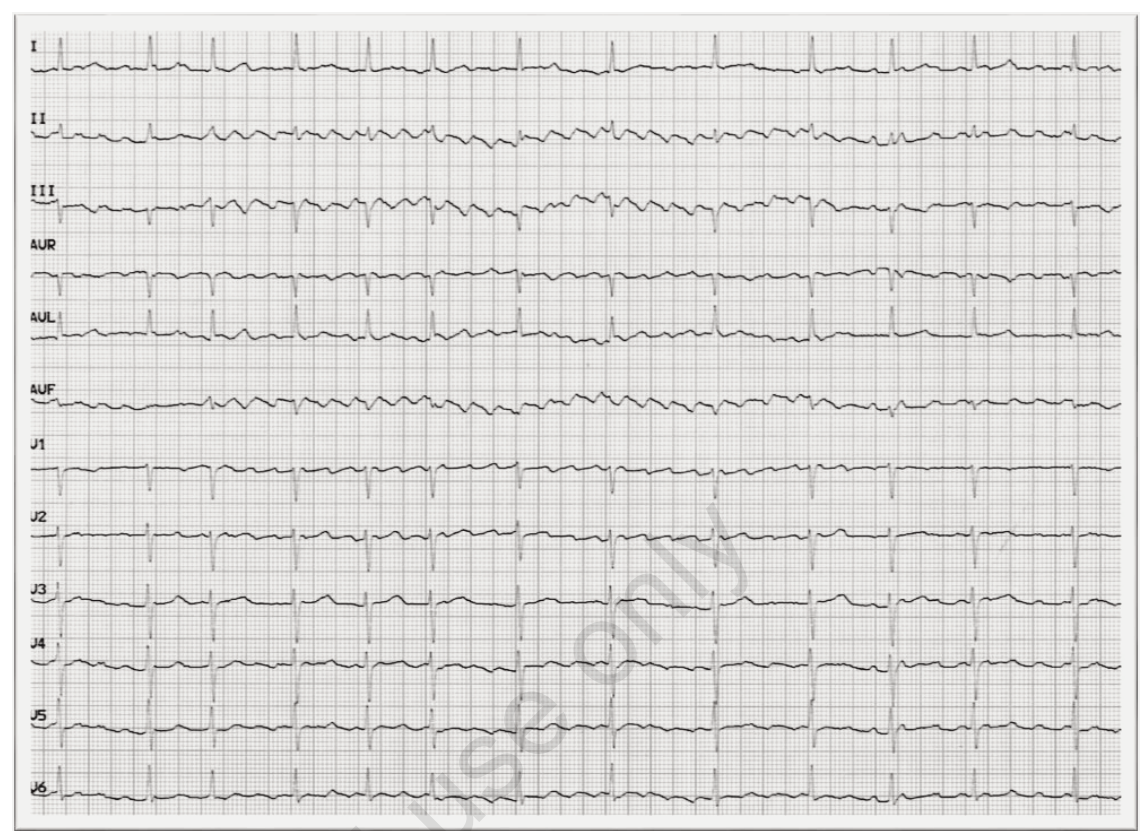

Figure 1. First ECG after resuscitation showing atrial fibrillation as the baseline rhythm.

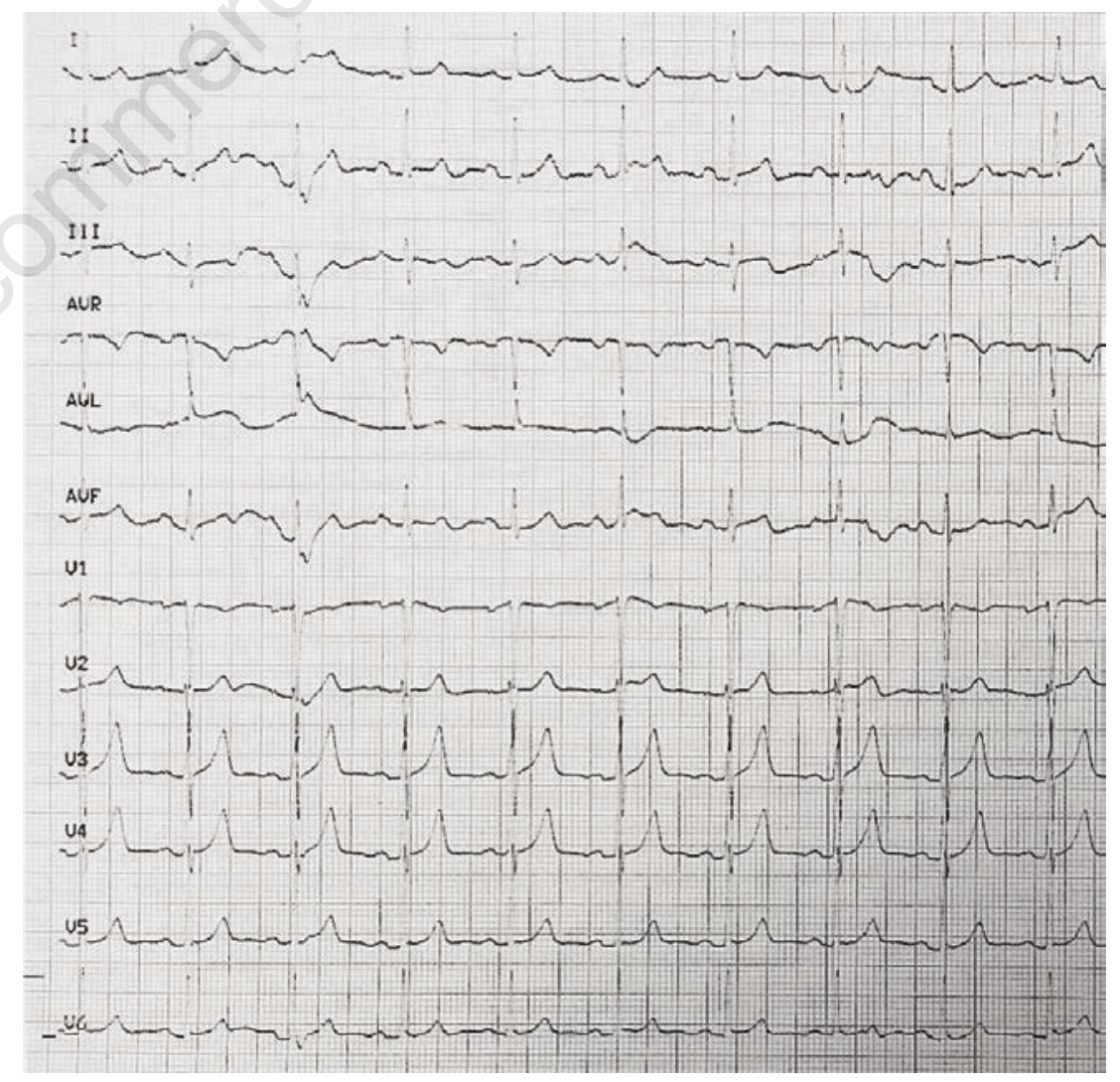

Figure 2. ECG after electrical cardioversion. The patient returned to sinus rhythm. QTc interval $423 \mathrm{~ms}$ (Bazett's formula). 


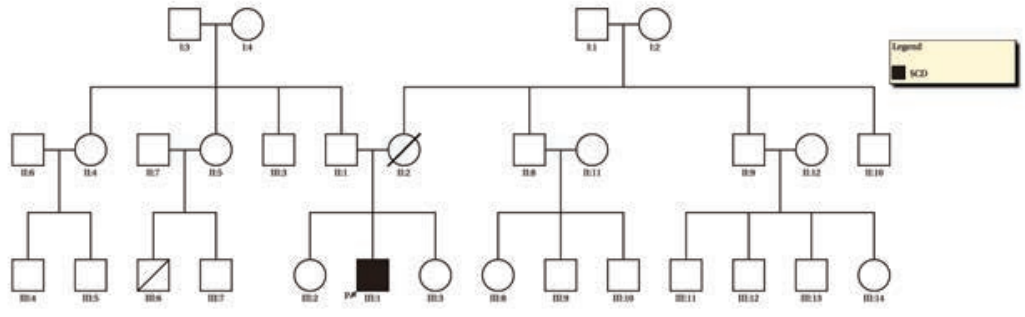

Figure 3. Pedigree of the family. The black arrow indicates the proband. Deceased family members are represented with single slanted line figures.

was associated with LQT1 and it was believed to produce a loss of function of the channel, ${ }^{6}$ functional analyses have shown that it indeed accelerates $\mathrm{I}_{\mathrm{Ks}}$ activation leading to an increased $\mathrm{K}^{+}$efflux (gain-of-function) during the repolarization. At atrial level, the subsequent shortening of the APD and refractoriness may promote reentrant arrhythmias and explain the AF phenotype of the carriers. ${ }^{7}$

The question is, however, how a gain of function mutation in KCNQ1 can lead in certain cases to LQTS or may produce a lethal VA even if the QT is not clearly prolonged. Tetrameric phosphorylation of Kv7.1 is required in order to enhance $I_{K s}$ current in response to $\beta$-adrenergic stimulation, a mechanism that is regulated by protein kinase A (PKA). ${ }^{13}$ Recent investigations have shown that some LQT1 mutations that are resistant to PKA activation are related to severe phenotypes with high risk of syncope and SCD. ${ }^{14}$ Computational modeling suggests that the $\mathrm{R} 231 \mathrm{H}$ mutation minimally affect the ventricular APD. Hence, a significant QT prolongation should not be expected in most of the carriers. However, functional analyses show that the mutation impairs the PKA-dependent phosphorylation of the Kv7.1channel. Therefore, in $\mathrm{R} 231 \mathrm{H}$ carriers, exercise and sympatheticinduced $\mathrm{I}_{\mathrm{Ks}}$ increase is prevented at ventricular level. The resistance to PKA phosphorylation might explain how a gain-of-function mutation might also cause abnormal ventricular repolarization under certain circumstances even when it does not produce a lengthening of the ventricular APD. ?

We believe that our case is in accordance to previous literature review. It seems that $\mathrm{R} 231 \mathrm{H}$ mutation unique properties lead to a very particular phenotype of early onset $\mathrm{AF}$ due to the increase $\mathrm{I}_{\mathrm{Ks}}$ current at atrial level. Though the QT interval can be upper limit or slightly prolonged, most patients do not present a typical LQTS but they still may have a significant risk of lethal arrhythmias because the ventricular excitability seems to be very abnormal. To the best of our knowledge, this is the second patient reported suffering cardiac arrest and
AF who carries the $\mathrm{R} 231 \mathrm{H}$ variant. As the case previously reported by Bartos, ${ }^{7}$ QT interval was not abnormally prolonged. An interesting observation in our opinion is that in both cases the SCD episode was at rest, a rare finding in KCNQ1 mutations since $\beta$ adrenergic stimulation is the main trigger for VA in LQT1 patients. The regulation of the $\mathrm{I}_{\mathrm{Ks}}$ channel is very complex and other molecules apart from PKA are implicated. Even if a misregulation in the PKA activation has been proposed as an explanation for ventricular abnormal repolarization in KCNQ1 R231H carriers, we think that further investigations are required to fully understand the underlying molecular mechanisms of such particular phenotype.

This case illustrates the importance of genetic testing as a tool in the diagnostic approach of unexplained SCD since genetic technologies can diagnose up to $27 \%$ of these unexplained cases. ${ }^{15}$ The identification of a pathogenic mutation and a deeper study of the effects of its expression besides the genotype itself might provide new insights into the understanding of inherited cardiac disorders.

\section{Conclusions}

A significant number of SCD especially in the young remain unexplained after a careful medical investigation. An inherited arrhythmogenic disorder should then be suspected. Genetic testing should be considered in the diagnostic workup in these cases. The identification of a pathogenic mutation and subsequent investigation of the molecular mechanisms underlying the phenotype might provide a personalized diagnosis, care and treatment to patients and their families.

\section{References}

1. Priori SG, Blomstrom-Lundqvist $\mathrm{C}$, Mazzanti A, et al. 2015 ESC Guidelines for the management of patients with ventricular arrhythmias and the prevention of sudden cardiac death: The Task Force for the Management of Patients with Ventricular Arrhythmias and the Prevention of Sudden Cardiac Death of the European Society of Cardiology (ESC). Endorsed by: Association for European Paediatric and Congenital Cardiology (AEPC). Eur Heart J 2015;36:2793-867.

2. Semsarian C, Ingles J, Wilde AA. Sudden cardiac death in the young: the molecular autopsy and a practical approach to surviving relatives. Eur Heart J 2015;36:1290-6.

3. Napolitano C, Priori SG, Schwartz PJ, et al. Genetic testing in the long QT syndrome: development and validation of an efficient approach to genotyping in clinical practice. JAMA 2005;294:2975-80.

4. Johnson JN, Tester DJ, Perry J, et al. Prevalence of early-onset atrial fibrillation in congenital long QT syndrome. Heart Rhythm 2008;5:704-9.

5. Kapplinger JD, Tester DJ, Salisbury BA, et al. Spectrum and prevalence of mutations from the first 2,500 consecutive unrelated patients referred for the FAMILION long QT syndrome genetic test. Heart Rhythm 2009;6:1297-303.

6. Guerrier K, Czosek RJ, Spar DS, Anderson J. Long QT genetics manifesting as atrial fibrillation. Heart Rhythm 2013;10:1351-3.

7. Bartos DC, Anderson JB, Bastiaenen R, et al. A KCNQ1 mutation causes a high penetrance for familial atrial fibrillation. J Cardiovasc Electrophysiol 2013;24:562-9.

8. Terrenoire C, Clancy CE, Cormier JW, et al. Autonomic control of cardiac action potentials: role of potassium channel kinetics in response to sympathetic stimulation. Circ Res 2005;96: e25-34.

9. Volders PG, Stengl M, van Opstal JM, et al. Probing the contribution of IKs to canine ventricular repolarization: key role for beta-adrenergic receptor stimulation. Circulation 2003;107:2753-60.

10. Jost N, Virag L, Bitay $M$, et al. Restricting excessive cardiac action potential and QT prolongation: a vital role for IKs in human ventricular muscle. Circulation 2005;112:1392-9.

11. Sanguinetti MC. Long QT syndrome: ionic basis and arrhythmia mechanism in long QT syndrome type 1. J Cardiovasc Electrophysiol 2000;11: 710-2.

12. Chen YH, Xu SJ, Bendahhou S, et al. KCNQ1 gain-of-function mutation in familial atrial fibrillation. Science 
2003;299:251-4.

13. Marx SO, Kurokawa J, Reiken S, et al. Requirement of a macromolecular signaling complex for beta adrenergic receptor modulation of the KCNQ1KCNE1 potassium channel. Science
2002;295:496-9.

14. Heijman J, Spatjens RL, Seyen SR, et al. Dominant-negative control of cAMP-dependent IKs upregulation in human long-QT syndrome type 1. Circ Res 2012;110:211-9.
15. Bagnall RD, Weintraub RG, Ingles J, et al. A prospective study of sudden cardiac death among children and young adults. N Engl J Med 2016;374:244152. 